\title{
Identification of Antihypertensive Tripeptides in the Culture Medium of Lactobacillus helveticus D75 and D76 Strains
}

\author{
Kirill Kuvakin 1,2, Timur Vakhitov 1,*, Stanislav Sitkin 1,4,5, Evgeniya Roshchina ${ }^{1}$, Veronika \\ Lisitskaya ${ }^{1}$, Olga Ivanchenko ${ }^{2}$, Yaroslav Dubrovsky ${ }^{3,4}$ and Elena Demyanova ${ }^{1}$ \\ 1 State Research Institute of Highly Pure Biopreparations of FMBA of Russia, 197110 Saint Petersburg, \\ Russia; alladinkira@mail.ru (K.K.); sitkins@yandex.ru (S.S.); roshek@yandex.ru (E.R.); vilisa53@yandex.ru \\ (V.L.); lenna_22@mail.ru (E.D.) \\ 2 Peter the Great St. Petersburg Polytechnic University, 195251 Saint Petersburg, Russia; \\ obivanchenko@yandex.ru \\ 3 Saint Petersburg State University, 199034 Saint Petersburg, Russia; dubrovskiy.ya@gmail.com \\ 4 Almazov National Medical Research Centre, 197341 Saint Petersburg, Russia \\ 5 North-Western State Medical University named after I.I. Mechnikov of the Ministry of Health of the \\ Russia, 191015 Saint Petersburg, Russia \\ * Correspondence: tim-vakhitov@yandex.ru
}

Published: 30 October 2020

\begin{abstract}
The aim of the current work was to search for bioactive peptides Ile-Pro-Pro (IPP) and Val-Pro-Pro (VPP) with antihypertensive action in the culture medium (CM) of probiotic Lactobacillus helveticus D75 and D76 strains when cultured in milk. The CM composition was analyzed using HPLC-UV, HPLC-MS, and MALDI Fourier transformation cyclotron resonance mass spectrometry (MALDI FT-ICR MS, Varian). Direct analysis of CM using FT-ICR failed to detect peptides in the original $\mathrm{CM}$ due to the high concentration of concomitant components, but after preliminary fractionation of CM by HPLC, both peptides were detected. The masses $(\mathrm{m} / \mathrm{z})$ of characteristic ions were 312.19 (VPP) and 326.21 (IPP). Peptides were quantified using a single reference method with HPLC and time-of-flight mass spectrometry. The concentrations of VPP and IPP in the CM of the D75 strain, respectively, reached $18.0 \mu \mathrm{g} / \mathrm{mL}$ and $25.4 \mu \mathrm{g} / \mathrm{mL}$, in the CM of the D76 strain they were lower $(12.7 \mu \mathrm{g} / \mathrm{mL}$ and $16.2 \mu \mathrm{g} / \mathrm{mL}$ ), and in the mixed CM (both strains) the concentrations reached intermediate values of $15.7 \mu \mathrm{g} / \mathrm{mL}$ and $24.0 \mu \mathrm{g} / \mathrm{mL}$. The data obtained indicate the existence of an active complex of extracellular enzymes in L. helveticus D75 and D76, which provide the production of IPP and VPP peptides with antihypertensive effect. The results also confirm the symbiotic properties of D75 and D76 strains (synergism and syntrophy). Functional foods based on milk fermented by probiotic L. helveticus D75 and D76 strains, which contains IPP and VPP peptides in effective doses $(>3.0 \mathrm{mg} /$ day), can be considered a safe and effective nonpharmacological intervention in patients with mild hypertension.
\end{abstract}

Keywords: Lactobacillus helveticus D75 and D76; antihypertensive effect; bioactive peptides; valylprolyl-proline; isoleucyl-prolyl-proline; fermented milk; probiotics; proteolytic activity; functional foods

\section{Introduction}

Lactobacillus helveticus D75 and D76 have been used as components of the Vitaflor probiotic dietary supplement since 1997. The combination of two closely related strains in one probiotic product is explained by their pronounced symbiotic relationships (synergy). When strains are grown 
together, the titer of viable bacteria increases, their resistance to stress increases, and their antagonistic activity against pathogens increases. The pronounced probiotic activity of strains and particularly their combinations have been confirmed by numerous clinical studies [1].

L. helveticus D75 and D76 strains have demonstrated efficacy in the treatment of chronic nasopharyngeal diseases, eradication of Helicobacter pylori infection in children with gastroduodenal lesions, treatment of mucosal lymphoma associated with lymphoid tissue (in cases where antibiotic treatment was ineffective), treatment of vulvovaginitis in girls, treatment of cancer patients with complications caused by chemotherapy and radiation therapy, treatment of infections caused by Klebsiella spp., and in the treatment of inflammatory bowel diseases [1,2].

The ability to utilize milk proteins is a fundamental property of probiotic lactic acid bacteria. By breaking down proteins using extracellular enzymes, lactobacilli primarily obtain a substrate for their own nutrition. However, biologically active peptides can be formed that have a positive effect on the host body.

Such peptides, for example, include the tripeptides Isoleucyl-Prolyl-Proline (IPP, Ile-Pro-Pro) and Valyl-Prolyl-Proline (VPP, Val-Pro-Pro), which inhibit the angiotensin-converting enzyme (ACE) and have antihypertensive properties, respectively [3]. In addition to these tripeptides, antihypertensive activity in experimental animal studies has been demonstrated by other peptides formed by various strains of $L$. helveticus. For example, L. helveticus CPN4 forms the Tyr-Pro dipeptide [4], and the strain L. helveticus CP790 is a series of peptides with a longer chain length: Ala-Tyr-PheTyr-Pro-Glu, Ser-Lys-Val-Leu-Pro-Val-Pro-Glu, and Lys-Val-Leu-Pro-Val-Pro-Gln [5].

The results of analysis of genome-wide sequencing data showed that the genomes of the $L$. helveticus D75 and D76 strains contain genes of such proteases such as lactocepin H3 (prtH3) and lactocepin $\mathrm{H}(p r t H)$, which are key enzymes in milk casein utilization [2]. It is also known that at least one of these, lactocepin $\mathrm{H} 3$, is involved in the formation of biologically active tripeptides IPP and VPP. This study was conducted to confirm the possibility of producing these peptides when cultivating L. helveticus D75 and D76 strains in milk.

\section{Materials and Methods}

2.1. Strains of bacteria. We used probiotic strains of Lactobacillus helveticus D75 and Lactobacillus helveticus D76 (commercial strains from Vitaflor dietary supplement, Russia) from the collection of the State Research Institute of Highly Pure Biopreparations of the Federal Medical-Biological Agency (FMBA) of Russia.

2.2. Cultivation of bacteria. Lactobacillus cultures were pre-activated for 2 passages, and then grown on milk in pure or mixed cultures. At the first stage, a tablet of freeze-dried L. helveticus cultures containing $2 \times 10^{8}$ bacteria were diluted in $2 \mathrm{~mL}$ of sterile water and rehydrated for $0.5 \mathrm{~h}$ at $37^{\circ} \mathrm{C}$, after which $8 \mathrm{~mL}$ of sterile milk was added, mixed, and incubated at $37^{\circ} \mathrm{C}$ for $21 \pm 2 \mathrm{~h}(1 \mathrm{st}$ passage). The resulting culture of the 1 st passage was introduced $(3 \% \mathrm{v} / \mathrm{v})$ into fresh sterile milk and also incubated at $37^{\circ} \mathrm{C}$ for $21 \pm 2 \mathrm{~h}$ (2nd passage). Similarly, an experimental culture of the 3rd passage was grown, from which samples were taken during the process (10 and $23 \mathrm{~h})$ to analyze the composition of the media. In experiments with mixed cultures, the first 2 passages were performed with pure D75 and D76 cultures, and for the 3rd passage, two volumes ( $2 \% \mathrm{v} / \mathrm{v})$ of D76 culture and one volume of D75 culture ( $1 \% \mathrm{v} / \mathrm{v})$ were seeded in accordance with the Vitaflor formulation.

2.3. Preparation of samples for chromatographic analysis. Ultrafiltrates of culture medium (UCM) for subsequent analysis were obtained by sequential centrifugation of CM samples taken during crop cultivation (20 min, 15,000 rpm, J2-21 centrifuge, Beckman (USA)) and their ultrafiltration through the Sartorius Hydrosart 10 membrane with a nominal molecular weight cutoff of $10 \mathrm{KD}$. The resulting $\mathrm{UCM}$ was stored at $20^{\circ} \mathrm{C}$.

2.4. MALDI-mass spectrometric analysis was performed on a Fourier transform ion-cyclotron resonance (FT-ICR) mass spectrometer (FT(ICR)MS, Varian, USA). A universal matrix, 2,5dihydroxybenzoic acid (DHB), was used for analysis. A quantity of $100 \mu \mathrm{L}$ of CS ultrafiltrate or its fraction was freeze-dried and dissolved in $1 \mu \mathrm{L}$ of matrix solution $(20 \mathrm{mg} / \mathrm{mL}$ in a mixture of acetonitrile:water:TFC (50:50:0.01\%)) and applied to the target. 
2.5. HPLC was performed on an LC-20 Prominence chromatograph (Shimadzu, Japan) with UV registration at $206 \mathrm{~nm}$ on a Luna C18, $5 \mu \mathrm{m}, 100 \AA, 250 \times 4.6 \mathrm{~mm}$ column (Phenomenex, USA) with a Supelguard H HPLC column $40 \times 4.6 \mathrm{~mm}$ (Sigma-Aldrich, USA). Using an acetonitrile gradient from $3 \%(0-1 \mathrm{~min})$ to $50 \%(23 \mathrm{~min}), \mathrm{t}=45{ }^{\circ} \mathrm{C}, \mathrm{V}=1 \mathrm{~mL} / \mathrm{min}$, and registration by optical density at a wavelength of $206 \mathrm{~nm}$, the volume of the injected sample is $20 \mu \mathrm{l}$. For MALDI-mass spectrometric analysis, fractions of 6.5-7.5 $\mathrm{min}$ (VPP) and 7.5-8.5 $\mathrm{min}$ (IPP) were collected.

2.6. HPLC-MS. HPLC-MS was performed using an Elute chromatographic system (Bruker, Germany) with a combined quadrupole-time-of-flight mass-selective maXis Impact detector (Q-TOF, Bruker, Germany) with electrospray ionization at atmospheric pressure under the following conditions: the flow of the desiccant gas (nitrogen) was $81 / \mathrm{min}$, the gas pressure on the nebulizer was 2 bar, the temperature of the conducting capillary was $220^{\circ} \mathrm{C}$, the voltage on the capillary was 4500 V. Chromatographic separation was performed on a column Intensity Solo 2 C18 (Bruker, Germany) $100 \times 2.1 \mathrm{~mm}$, particle diameter 1.8 microns, pore size $90 \AA$ A . Elution was performed in a gradient system of $0.1 \%$ formic acid-acetonitrile with an acetonitrile gradient of 15 to $85 \%$. Eluent flow rate$0.3 \mathrm{~mL} / \mathrm{min}$; column temperature $-50{ }^{\circ} \mathrm{C}$; auto - sampler temperature $-10{ }^{\circ} \mathrm{C}$; sample volume $-1 \mu \mathrm{l}$; analysis time $-5 \mathrm{~min}$.

2.7. Reference IPP and VPP peptides were synthesized in the Laboratory of Peptide Chemistry State Research Institute of Highly Pure Biopreparations of FMBA of Russia (Dr. A. A. Kolobov and Dr. M. P. Smirnova).

\section{Results and Discussion}

This work was devoted to the search for biologically active tripeptides VPP and IPP. The purity of the peptides was confirmed by HPLC in a gradient system of acetonitrile and $0.1 \%$ trifluoroacetic acid (TFC). The obtained retention times of VPP and IPP were 6.84 and $8.05 \mathrm{~min}$, respectively. These values were used as reference points for UCM fractionation, which was carried out in the same way. The structure of the peptides was confirmed by MALDI-mass spectrometric analysis. Their defined molecular weights were 312.1927 (VPP) and 326.2086 (IPP). Initially, an attempt was made to determine by this method the presence of the desired tripeptides in culture fluids, but it was not successful due to the complexity of the composition of the media under study. If the media were subjected to preliminary fractionation by HPLC, the desired peptides were determined by this method in the same fractions as the reference peptides (Figure 1).

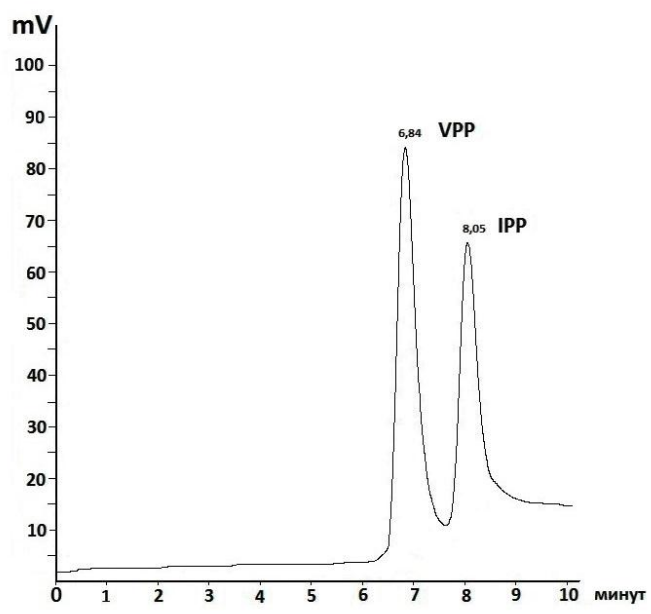

Figure 1. HPLC of a mixture of Val-Pro-Pro (VPP) and Ile-Pro-Pro (IPP) standards. The column was applied to $20 \mathrm{~mL}$ of a solution of IPP and VPP with a concentration of $75 \mathrm{mg} / \mathrm{mL}$ each. The column was a phenomenex Luna C18. Eluent of $0.1 \%$ TFC with an acetonitrile gradient from $3 \%(0-1 \mathrm{~min})$ to $50 \%(23 \mathrm{~min}), \mathrm{t}=45^{\circ} \mathrm{C}, \mathrm{V}=1 \mathrm{~mL} / \mathrm{min}$, registration by optical density at a wavelength of $206 \mathrm{~nm}$. 
The figures show that compounds with masses corresponding to the desired peptides are found in the fractions at 6.5-7.5 $\mathrm{min}$ (VPP) and 7.5-8.5 $\mathrm{min}$ (IPP). Thus, in the culture medium of the $L$. helveticus D75 strain, both peptides were found. The accuracy of matching the masses with the masses of synthetic peptides was at least 0.001 a.w.u. Similar data were obtained for L. helveticus D76.

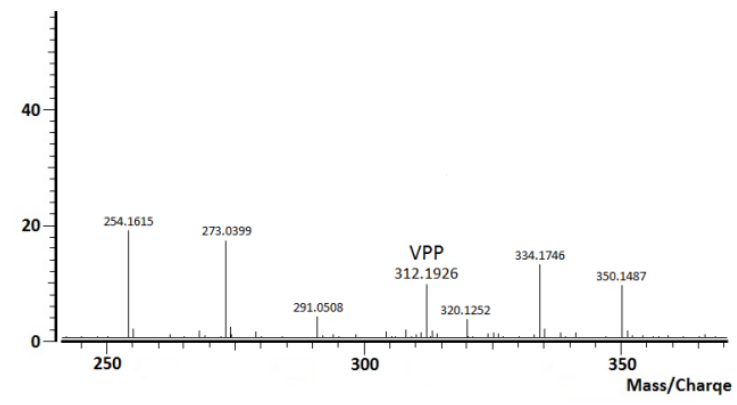

(a)

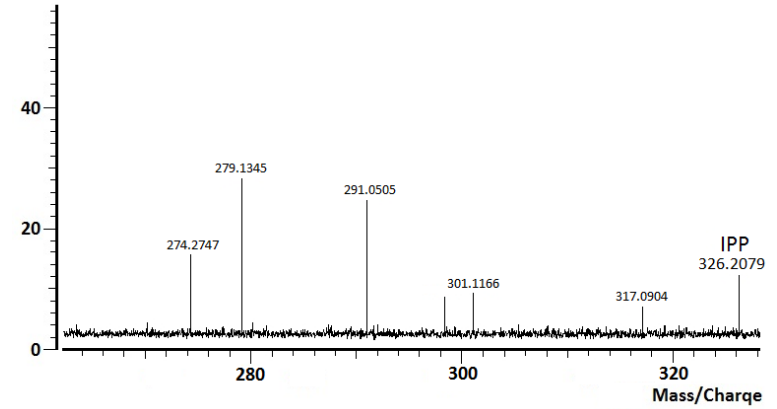

(b)

Figure 2. MALDI-mass spectrometric analysis of fractions of culture medium (CM) ultrafiltrates of the L. helveticus D75 strain. The mass spectra were obtained using a Varian ion-cyclotron resonance (ICR) mass spectrometer. The relative intensity is plotted on the ordinate, and the mass-to-charge ratio $(\mathrm{m} / \mathrm{z})$ is plotted on the abscissa. (a), (b) UCM fractions: $24 \mathrm{~h}$ of growth of the L. helveticus D75 strain: (a) fractions of 6.5-7.5 $\mathrm{min}$; (b) fractions of 7.5-8.5 min.

To determine the concentrations of peptides in the culture media of L. helveticus D75 and D76, an HPLC-MS analysis was performed. The results of the analysis are shown in Figure 3, and the description of the gradient system of the eluent is provided in Table 1.

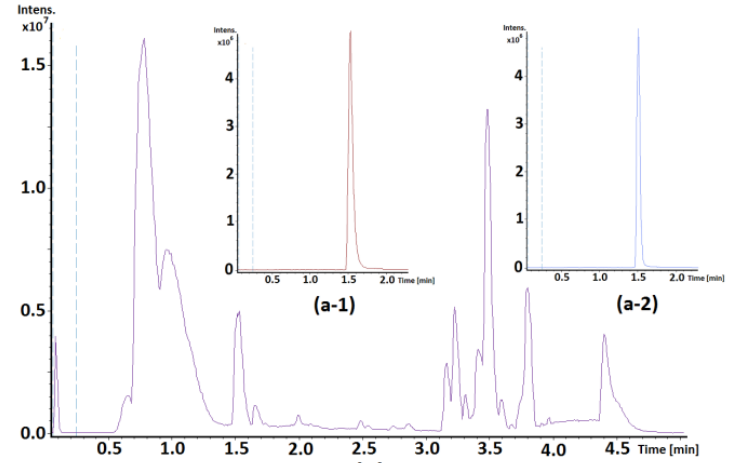

(a)

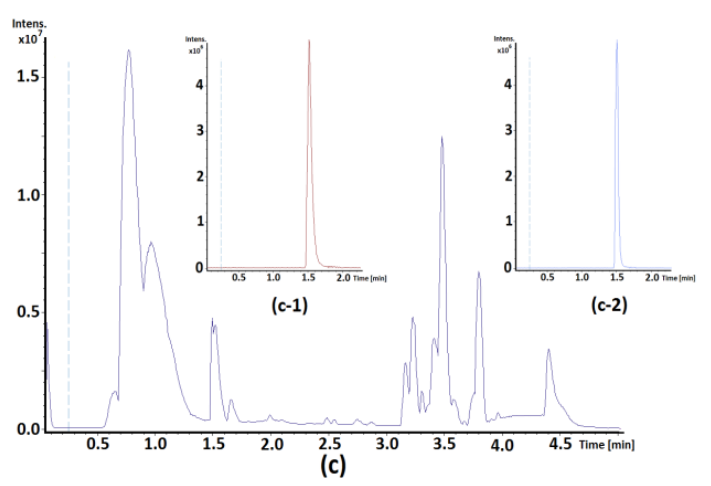

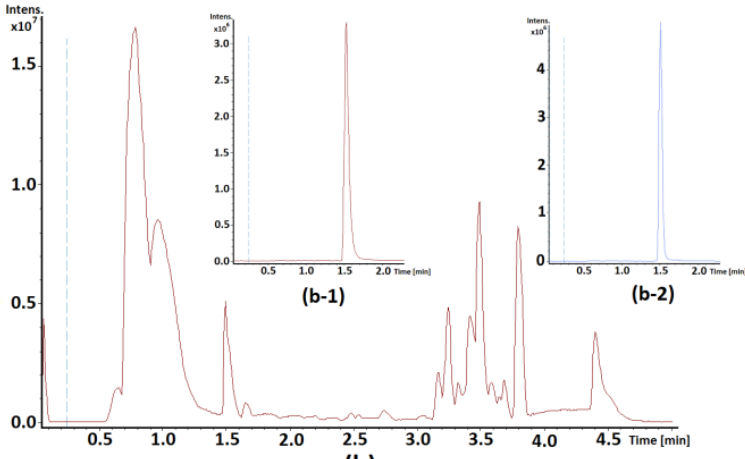

(b)

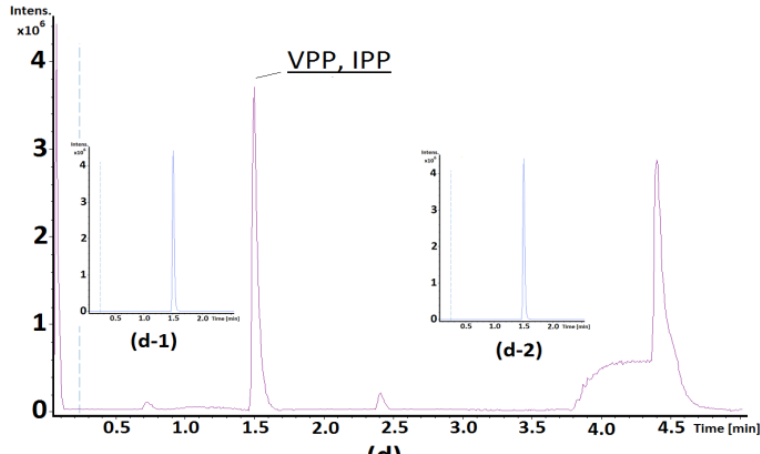

(d)

Figure 3. HPLC-MS of CM ultrafiltrates on an Intensity Solo 2C 18 column in a gradient system of $0.1 \%$ formic acid-acetonitrile (Table 1). Relative intensity is plotted on the ordinate, and time in minutes is plotted on the abscissa. (a-d) - registration of total ion current: (a) L. helveticus D75, (b) L. helveticus D76, (c) mixed culture of D75 and D76 strains, and (d) a mixture of synthetic peptides of IPP and VPP in concentrations of 10 micrograms $/ \mathrm{mL}$. In the insets to the figures, registration of chromatographic 
separation by ion current of characteristic ions with $\mathrm{m} / \mathrm{z}=326.21$ (IPP) $-(\mathrm{a}-1, \mathrm{~b}-1, \mathrm{c}-1, \mathrm{~d}-1)$ and 312.19 $(\mathrm{VPP})-(\mathrm{a}-2, \mathrm{~b}-2, \mathrm{c}-2, \mathrm{~d}-2)$.

Table 1. A gradient eluent system for the quantification of the tripeptides VPP and IPP by HPLC-MS.

\begin{tabular}{ccc}
\hline $\mathbf{t}, \mathbf{m i n}$ & $\mathbf{0 . 1 \%}$ of Formic Acid & $\mathbf{1 0 0 \%} \mathbf{A C N}$ \\
\hline $0 \rightarrow 1.5$ & $85 \%$ & $15 \%$ \\
$1.5 \rightarrow 2.5$ & $85 \% \rightarrow 65 \%$ & $15 \% \rightarrow 35 \%$ \\
\hline $2.5 \rightarrow 2.7$ & $65 \% \rightarrow 20 \%$ & $35 \% \rightarrow 80 \%$ \\
\hline $2.7 \rightarrow 3.3$ & $20 \%$ & $80 \%$ \\
\hline $3.3 \rightarrow 3.5$ & $20 \% \rightarrow 85 \%$ & $80 \% \rightarrow 15 \%$ \\
\hline $3.5 \rightarrow 5$ & $85 \%$ & $15 \%$ \\
\hline
\end{tabular}

Table 2 shows that the retention times of peptides in this separation method of IPP and VPP were almost identical. However, the presence of both peptides is clearly visible in the insets of the figure when recording the separation of characteristic ions (326.21 (IPP) and 312.19 (VPP)) by ion current. Thus, it is clearly visible that both strains have a pronounced ability to break down milk casein to form peptides with an antihypertensive effect. In addition, the D75 strain produces approximately 1.4 times more of the VPP peptide and 1.6 times more of the IPP peptide. When strains are grown together, the level of tripeptides is slightly lower than in the culture medium of the most productive of these (D75), but significantly higher than in the medium of the strain (D76).

Table 2. Concentrations of peptides in the culture fluid, according to HPLC-MS data, during the cultivation of lactobacilli in milk for $23 \mathrm{~h}$.

\begin{tabular}{ccccc}
\hline \multirow{2}{*}{ Sample } & \multicolumn{2}{c}{ Retention Time, min } & \multicolumn{2}{c}{ Concentration, $\boldsymbol{\mu g} / \mathbf{m l}$} \\
\cline { 2 - 5 } & VPP & IPP & VPP & IPP \\
\hline D75 & 1.52 & 1.54 & 18.0 & 25.4 \\
\hline D76 & 1.52 & 1.53 & 12.7 & 16.2 \\
\hline D75 + D76 & 1.52 & 1.52 & 15.7 & 24.0 \\
\hline IPP, VPP & 1.52 & 1.52 & 10.0 & 10.0 \\
\hline
\end{tabular}

It is interesting to compare the concentrations of peptides in milk fermented with strains D75 and D76 with their minimum effective dose, which according to available data is about $3.07 \mathrm{mg}$ per day [3]. As can be seen from Table 2, the daily norm of both peptides contains approximately $170 \mathrm{~mL}$ of milk fermented with the D75 strain, and $242 \mathrm{~mL}$ of milk fermented with the D76 strain. If a commercial preparation is used for the starter culture, which is a mixture of both strains, the effective dose will be $196 \mathrm{~mL}$.

In terms of the practical significance of the study, it is worth noting that the identified tripeptides IPP and VPP have previously confirmed their antihypertensive effect not only in experimental, but also in clinical studies [6-9], which allows food products containing them as functional to be considered.

Hypertension, which is a significant risk factor for other diseases of the cardiovascular system, strokes, kidney failure, and cerebrovascular disorders, affects more than 1 billion people globally, according to the WHO [10]. Because long-term use of antihypertensive medications can lead to the development of side effects in a significant portion of patients, non-drug methods for reducing high blood pressure are constantly being sought [11]. One of the effective and safe options for non-drug correction could be the use of functional fermented milk products obtained by fermenting milk with sourdough based on probiotic strains of L. helveticus D75 and D76 and containing VPP and IPP in effective daily doses (>3.0 mg) [3,9]. It is possible that regular use of such products will allow some patients with mild forms of arterial hypertension (stage 1) to reduce the dose of antihypertensive drugs taken or even abandon these drugs.

In addition, experimental studies have shown that antihypertensive tripeptides IPP and VPP are able to suppress cytokine-mediated inflammatory responses in adipocytes, showing pronounced 
insulin-like activity [12,13]. Given the critical role of adipose tissue in the pathogenesis of hypertension and its relationship with inflammation and insulin resistance, functional products containing these tripeptides may be potentially effective in patients with various forms of metabolic syndrome [6,14]. Randomized clinical trials are required to confirm this assumption.

In conclusion, it can be noted that the fact that biologically active compounds produced by probiotic strains of L. helveticus D75 and D76 are detected in the culture fluid, taking into account the genetic potential of these microorganisms, allows us be optimistic that there will be experimental and clinical confirmation in subsequent studies of other beneficial effects for human health associated with their metabolic activity and the production of exometabolites [15]. In addition to the known effects, such as antagonistic activity against pathogenic microorganisms, including Candida, immunomodulatory action, and production of antihypertensive peptides $[5,14,16]$, probiotic bacteria L. helveticus, according to the results of recent scientific studies, can reduce the levels of cholesterol (cholesterol-lowering effect) and low-density lipoproteins in the blood (hypolipidemic effect), improve digestion ( $\alpha$ - and $\beta$-galactosidase activity), strengthen the mucosal barrier and suppress inflammatory processes in the intestine (anti-inflammatory effect), inhibit colorectal oncogenesis (antitumor effect), modulate microbial homeostasis (production of bacteriocins), provide antioxidant and anti-aging effects, and improve liver function when combined with appropriate diet and exercise $[5,17]$.

\section{References}

1. Vorob'ev, A.A.; Gershanovich, M.L.; Petrov, L.N. Prerequisites and perspectives for use of probiotics in complex therapy of cancer. Vopr. Onkol. 2004, 50, 361-365.

2. Toropov, V.; Demyanova, E.; Shalaeva, O.; Sitkin, S.; Vakhitov, T. Whole-Genome Sequencing of Lactobacillus helveticus D75 and D76 Confirms Safety and Probiotic Potential. Microorganisms 2020, 8, 329.

3. Jäkälä, P.; Vapaatalo, H. Antihypertensive Peptides from Milk Proteins. Pharmaceuticals 2010, 3, 251-272.

4. Yamamoto, N.; Maeno, M.; Takano, T. Purification and characterization of an antihypertensive peptide from a yogurt-like product fermented by Lactobacillus helveticus CPN4. J. Dairy Sci. 1999, 82, 1388-1393.

5. Taverniti, V.; Guglielmetti, S. Health-Promoting Properties of Lactobacillus helveticus. Front. Microbiol. 2012, 3, 392 .

6. Cicero, A.F.; Colletti, A.; Rosticci, M.; Cagnati, M.; Urso, R.; Giovannini, M.; Borghi, C.; D’Addato, S. Effect of Lactotripeptides (Isoleucine-Proline-Proline/Valine-Proline-Proline) on Blood Pressure and Arterial Stiffness Changes in Subjects with Suboptimal Blood Pressure Control and Metabolic Syndrome: A DoubleBlind, Randomized, Crossover Clinical Trial. Metab. Syndr. Relat. Disord. 2016, 14, 161-166.

7. Boelsma, E.; Kloek, J. IPP-rich milk protein hydrolysate lowers blood pressure in subjects with stage 1 hypertension, a randomized controlled trial. Nutr. J. 2010, 9, 52.

8. Cicero, A.F.; Aubin, F.; Azais-Braesco, V.; Borghi, C. Do the lactotripeptides isoleucine-proline-proline and valine-proline-proline reduce systolic blood pressure in European subjects? A meta-analysis of randomized controlled trials. Am. J. Hypertens. 2013, 26, 442-449.

9. Beltrán-Barrientos, L.M.; Hernández-Mendoza, A.; Torres-Llanez, M.J.; González-Córdova, A.F.; VallejoCórdoba, B. Invited review: Fermented milk as antihypertensive functional food. J. Dairy Sci. 2016, 99, 40994110.

10. Kintscher, U. The burden of hypertension. EuroIntervention 2013, 9, R12-R15.

11. Ozemek, C.; Laddu, D.R.; Arena, R.; Lavie, C.J. The role of diet for prevention and management of hypertension. Curr. Opin. Cardiol. 2018, 33, 388-393.

12. Chakrabarti, S.; Wu, J. Milk-derived tripeptides IPP (Ile-Pro-Pro) and VPP (Val-Pro-Pro) promote adipocyte differentiation and inhibit inflammation in 3T3-F442A cells. PLoS ONE 2015, 10, e0117492.

13. Chakrabarti. S.; Jahandideh, F.; Davidge, S.T.; Wu, J. Milk-Derived Tripeptides IPP (Ile-Pro-Pro) and VPP (Val-Pro-Pro) Enhance Insulin Sensitivity and Prevent Insulin Resistance in 3T3-F442A Preadipocytes. J. Agric. Food Chem. 2018, 66, 10179-10187.

14. Ricci-Cabello, I.; Herrera, M.O.; Artacho, R. Possible role of milk-derived bioactive peptides in the treatment and prevention of metabolic syndrome. Nutr. Rev. 2012, 70, 241-255. 
15. Vakhitov, T.Y.; Chalisova, N.I.; Sitkin, S.I.; Sall, T.S.; Shalaeva, O.N.; Demyanova, E.V.; Morugina, A.S.; Vinogradova, A.F.; Petrov, A.V.; Nozdrachev, A.D. Low-molecular-weight components of the metabolome control the proliferative activity in cellular and bacterial cultures. Dokl. Biol. Sci. 2017, 472, 8-10.

16. Avalueva, E.B.; Sitkin, S.I.; Tkachenko, E.I.; Uspenskij, J.P.; Shevjakov, M.A.; Dobritsa, V.P.; Zhigalova, T.N.; Skazyvaeva, E.V.; Nilova, L.J.; Petrov, L.N. Method of Treating Patients with Ulcerative Colitis. Russian Patent RU 2419443, 27 May 2011.

17. Fontana, A.; Falasconi, I.; Molinari, P.; Treu, L.; Basile, A.; Vezzi. A.; Campanaro, S.; Morelli, L. Genomic Comparison of Lactobacillus helveticus Strains Highlights Probiotic Potential. Front. Microbiol. 2019, 10, 1380.

Publisher's Note: MDPI stays neutral with regard to jurisdictional claims in published maps and institutional affiliations.

(C) 2020 by the authors. Licensee MDPI, Basel, Switzerland. This article is an open access article distributed under the terms and conditions of the Creative Commons Attribution (CC BY) license (http://creativecommons.org/licenses/by/4.0/). 